\title{
Matéria
}

ISSN 1517-7076

Revista Matéria, v. 12, n. 3, pp. 453 - 461, 2007

http://www.materia.coppe.ufrj.br/sarra/artigos/artigo10822

\section{Espectroscopia fototérmica e difração de raios x: Técnicas complementares para a investigação do $\mathrm{Cr}^{3+}$ no sistema $\mathrm{LiGaSiO}_{4}-\mathrm{LiGa}_{5} \mathrm{O}_{8}-\mathrm{Li}_{5} \mathrm{GaSi}_{2} \mathrm{O}_{8}$}

\author{
Pedro, S.S. ; Sosman, L.P. ${ }^{\mathrm{I}}$
}

Instituto de Física - Universidade do Estado do Rio de Janeiro

Rua São Francisco Xavier 524. Maracanã, Rio de Janeiro, RJ. CEP: 20559-900

e-mail: sspedro80@gmail.com, sosman@uerj.br

\section{RESUMO}

Neste trabalho tentamos estabelecer um método de preparo conveniente para a obtenção da fase $\mathrm{LiGaSiO}_{4}$ de forma predominante no sistema $\mathrm{LiGaSiO}_{4}-\mathrm{LiGa}_{5} \mathrm{O}_{8}-\mathrm{Li}_{5} \mathrm{GaSi}_{2} \mathrm{O}_{8}$ contendo $\mathrm{Cr}^{3+}$ como impureza substitucional. As amostras foram produzidas com os componentes $\mathrm{Li}_{2} \mathrm{CO}_{3}, \mathrm{Ga}_{2} \mathrm{O}_{3}, \mathrm{SiO}_{2}$ e $\mathrm{Cr}_{2} \mathrm{O}_{3}$. Inicialmente, os óxidos foram misturados mecanicamente durante $2 \mathrm{~h}$ em um gral de ágata e prensados em forma de pastilhas de $10 \mathrm{~mm}$ de diâmetro e $2 \mathrm{~mm}$ de espessura, sob carga de 2t. As pastilhas foram levadas ao forno e aquecidas até $1000^{\circ} \mathrm{C}$, dentro de cadinhos de alumina. Após $72 \mathrm{~h}$ o forno foi desligado e esta amostra de pastilhas foi resfriada dentro do forno. A amostra obtida foi investigada por difração de raios X para a verificação da proporção entre as fases formadas. Uma nova amostra, destinada ao objetivo de variar a proporção entre as fases, foi preparada moendo os óxidos durante $6 \mathrm{~h}$, pastilhados sob $2 \mathrm{t}$, e queimada também a $1000^{\circ} \mathrm{C}$, porém durante 90 h. Após este tempo de residência à temperatura de queima, o forno foi desligado, iniciando o processo de resfriamento da amostra dentro do mesmo. Em seguida realizamos as medidas de espectroscopia fototérmica nas pastilhas das duas amostras. $O$ interesse na pesquisa desses materiais se justifica pelo fato de que eles apresentam bandas largas de absorção e emissão, nas regiões espectrais do visível e do infravermelho próximo. Estas bandas são sintonizáveis e permitem um amplo número de aplicações: em medicina, comunicações e lasers de estado sólido, por exemplo.

Palavras chaves: Cerâmicas, $\mathrm{Cr} 3+$, espectroscopia fototérmica.

\section{Photothermal spectroscopy and x-ray diffraction: Complementary techniques for $\mathrm{Cr}^{3+}$ investigation in $\mathrm{LiGaSiO}_{4}-\mathrm{LiGa}_{5} \mathrm{O}_{8}-\mathrm{Li}_{5} \mathrm{GaSi}_{2} \mathrm{O}_{8}$ system ABSTRACT}

In this work we tried to establish a convenient ceramics preparation method to obtain $\mathrm{LiGaSiO}_{4}$ phase as the predominant one in $\mathrm{LiGaSiO}_{4}-\mathrm{LiGa}_{5} \mathrm{O}_{8}-\mathrm{Li}_{5} \mathrm{GaSi}_{2} \mathrm{O}_{8}$ system containing few $\mathrm{Cr}^{3+}$ ions as substitutional impurity. The powder samples were produced by solid state reaction from high purity $\mathrm{Li}_{2} \mathrm{CO}_{3}, \mathrm{Ga}_{2} \mathrm{O}_{3}, \mathrm{SiO}_{2}$ and $\mathrm{Cr}_{2} \mathrm{O}_{3}$ powders. At first, the oxides were mixed during $2 \mathrm{~h}$ in an agate mortar and pressed into discs with dimensions $10 \mathrm{~mm}$ in diameter and $2 \mathrm{~mm}$ in thickness. The discs were fired in air for $72 \mathrm{~h}$ at $1000^{\circ} \mathrm{C}$ under atmospheric pressure. This first sample of discs was cooled down inside the furnace. With the objective of varying the ratio between the phases, a new sample was prepared by $6 \mathrm{~h}$ milling followed by pressing into discs with $2 \mathrm{t}$ charge and $90 \mathrm{~h}$ firing at $1000^{\circ} \mathrm{C}$. The two samples obtained were investigated by $\mathrm{X}$-ray diffraction, for determining the phases formed. Finally, photothermal spectroscopy measurements were carried out. The broad absorption and emission bands in the visible and near-infrared spectral regions presented by these materials constitute the motivation for their study. These bands are tunable and allow a large number of applications: in medicine, communications and solid state lasers, for example.

Keywords: Ceramics, $\mathrm{Cr}^{3+}$, photothermal spectroscopy.

\section{INTRODUÇÃO}

Materiais óxidos contendo íons de transição como impurezas substitucionais podem apresentar largas bandas de absorção e/ou emissão nas regiões espectrais do visível e do infravermelho próximo. Estas redes 
hospedeiras são formadas por íons de camadas fechadas, dentre os quais deve haver um íon de mesma valência e com raio iônico de valor próximo ao do íon impureza, para que a substituição seja facilitada. Entretanto, não é incomum a utilização de redes hospedeiras que não satisfaçam a estes requisitos. Neste caso, as diferenças entre as valências ou entre os raios iônicos são compensadas por posicionamentos dos íons hospedeiros na rede durante o processo de síntese. Quando o íon impureza é inserido na rede, os seus níveis eletrônicos são desdobrados em diferentes configurações eletrônicas pelo potencial eletrostático gerado pelos íons vizinhos (campo cristalino). As transições entre estados de diferentes configurações originam as largas bandas observadas, conhecidas como bandas vibracionais. Assim sendo, materiais óxidos dopados com metais de transição são candidatos para aplicações que variam de lasers de estado sólido a temperatura ambiente até telas de vídeo ("displays") de alta definição [1] . Materiais contendo $\mathrm{Cr}^{3+}$ em sítios de coordenação octaédricas são bastante estudados na literatura e utilizados como meio ativo de lasers de estado sólido, como por exemplo, $\mathrm{Be}_{3} \mathrm{Al}_{2}\left(\mathrm{SiO}_{3}\right)_{6}$ [2], $\mathrm{LiCaAlF}_{6}$ [3], $\mathrm{LiSrAlF}_{6}$ [4] e o $\mathrm{La}_{3} \mathrm{Ga}_{5} \mathrm{SiO}_{14}$ [5]. Porém materiais contendo o $\mathrm{Cr}^{3+}$ em sítios de coordenação tetraédrica são pouco estudados. Recentemente estudos de EPR e fotoluminescência associaram propriedades ópticas e magnéticas do $\mathrm{LiAlO}_{2}: \mathrm{Cr}^{3+}$ com a ocupação do $\mathrm{Cr}^{3+}$ em sítios tetraédricos do $\mathrm{Al}^{3+}$ [6]. Este fato justifica o interesse na produção e análise da fase pura do $\mathrm{LiGaSiO}_{4}$, no qual todos os íons são coordenados tetraedricamente [7].

\section{MATERIAIS E MÉTODOS}

As amostras foram preparadas a partir dos óxidos $\mathrm{PA} \mathrm{Li}_{2} \mathrm{CO}_{3}, \mathrm{Ga}_{2} \mathrm{O}_{3}, \mathrm{SiO}_{2}$ e $\mathrm{Cr}_{2} \mathrm{O}_{3}$. A quantidade de cada componente foi calculada para que obtivéssemos dois gramas de composto estequiométrico de $\mathrm{LiGaSiO}_{4}$, com $5 \%$ de $\mathrm{Cr}^{3+}$. Na produção da amostra 1, os óxidos foram misturados mecanicamente em um gral de ágata com pistilo, durante 2 horas. A amostra foi prensada na forma de pequenas pastilhas circulares com $10 \mathrm{~mm}$ de diâmetro e $2 \mathrm{~mm}$ de espessura, sob duas toneladas. As pastilhas foram colocadas em cadinhos de alumina e queimadas a $1000^{\circ} \mathrm{C}$ durante 72 horas em ar atmosférico. Após o resfriamento no próprio forno, a amostra foi pulverizada no gral de ágata até estar na forma de um pó bem fino, para a realização da difração de raios-X e espectroscopia fototérmica. Após as análises, uma nova amostra de pastilhas (2) foi preparada, homogeneizada mecanicamente por 6 horas, pastilhada sob duas toneladas e queimadas no forno a $600^{\circ} \mathrm{C}$ durante $1 \mathrm{~h}$ e $30 \mathrm{~min}$ seguida de continuação da queima a $1000^{\circ} \mathrm{C}$ durante 88 horas e $30 \mathrm{~min}$. Ao término deste intervalo de tempo o forno foi desligado e a amostra sofreu o resfriamento por inércia, sendo então retirada do forno e pulverizada para a realização da difração de raios $\mathrm{X}$ e da espectroscopia fototérmica. As pastilhas apresentaram aspecto homogêneo e cor verde, característica da valência 3+ do cromo. O grande problema enfrentado no estudo dos compostos óxidos é a sua obtenção. A qualidade da reação de estado sólido em misturas estequiométricas dependerá de grandezas tais como a temperatura do meio, o tempo do tratamento térmico e a evolução temporal da temperatura, o gradiente de temperatura dentro do forno, a energia de ativação da reação, o tamanho das partículas componentes e a estrutura cristalina dos reagentes. Dependendo da composição e do tratamento térmico, o produto final pode possuir várias fases cristalinas. O método aqui apresentado foi escolhido de acordo com o diagrama de fases de soluções sólidas contendo o $\mathrm{LiGaSiO}_{4}$ [ㅈ].

A difração de raios $\mathrm{X}$ foi realizada com um difratômetro de pó Siemens tipo $\mathrm{F}$ equipado com tubo Philips $2273 / 20(\lambda=1,542 \AA)$ alimentado a $35 \mathrm{kV}$ e $25 \mathrm{~mA}$, com monocromador de grafite e um detector Siemens de NaI acoplado à cavidade. A contagem foi em 2,0 segundos para cada passo de $0,05^{\circ}$, no intervalo de varredura entre $10^{\circ}$ e $70^{\circ}$. Após as medidas de raios $\mathrm{X}$, as amostras foram investigadas com a técnica de espectroscopia fototérmica a temperatura ambiente.

As medidas de fototérmica foram realizadas com uma fonte de luz policromática (lâmpada de xenônio de $800 \mathrm{~W}$ ) modulada em amplitude a $80 \mathrm{~Hz}$ por um modulador eletromecânico (Stanford Research System, modelo SR 540), que envia um sinal de referência para o amplificador síncrono (lock-in, Stanford Research System, modelo SR 830). Esta luz passa pelo monocromador que separa a luz branca em suas diversas componentes, obtendo-se luz monocromática. A luz atinge então a amostra, gerando o sinal fotoacústico que é captado por um microfone B\&K, no interior da célula. Este sinal é enviado para o amplificador, que amplifica e retifica as componentes do sinal que possuem a mesma freqüência de modulação do sinal de referência, eliminando sinais espúrios e indesejáveis. A seguir o sinal é enviado para um microcomputador onde é processado e analisado. O espectro fototérmico, portanto, é um gráfico de amplitude do sinal acústico em função do comprimento de onda que atinge a amostra. Como a lâmpada emite cada comprimento de onda com uma intensidade diferente, os dados obtidos para a amostra são normalizados em relação ao espectro de emissão da lâmpada. 


\section{RESULTADOS EXPERIMENTAIS E DISCUSSÃO}

\subsection{Difração de Raios X}

A primeira técnica de análise utilizada foi a difração de raios $\mathrm{X}$. O difratograma é um gráfico da intensidade da radiação espalhada em função do ângulo de espalhamento. Com os valores dos ângulos de cada um dos máximos de intensidade e utilizando um programa de cálculo simples e a lei de Bragg [9] são determinadas as correspondentes distâncias interplanares $d$, que identificam a estrutura cristalográfica. Com os valores calculados para as distâncias interplanares e partindo de uma família de compostos que poderiam ser formados a partir dos óxidos primários, identificamos a formação de três fases: aquela desejada, $\mathrm{LiGaSiO}_{4}$, acompanhada das fases $\mathrm{LiGa}_{5} \mathrm{O}_{8}$ e $\gamma-\mathrm{Li}_{5} \mathrm{GaSi}_{2} \mathrm{O}_{8}$. Os resultados obtidos para as amostras 1 e 2 são exibidos na Figura 1 (a) e (b), respectivamente.


Figura 1: Difratograma de pó (a) amostra 1 e (b) amostra 2, identificadas como misturas das fases $\mathrm{LiGaSiO}_{4}, \mathrm{LiGa}_{5} \mathrm{O}_{8}$ e $\gamma-\mathrm{Li}_{5} \mathrm{GaSi}_{2} \mathrm{O}_{8}$ em diferentes proporções.

Nas Tabelas 1 e 2 estão relacionadas as linhas de difração mais intensas identificadas, obtidas nas medidas das amostras 1 e 2, respectivamente, e as distâncias interplanares $d$. Os valores são comparados com a base JCPDS $[\underline{10}, \underline{11}]$ e com dados da literatura []]. 
Tabela 1: Linhas de difração mais intensas obtidas para a amostra 1.

\begin{tabular}{|c|c|c|c|c|c|c|c|}
\cline { 2 - 8 } \multicolumn{2}{c|}{} & \multicolumn{6}{c|}{ Valores Tabelados } \\
\hline \multicolumn{2}{|c|}{ Valores Medidos } & \multicolumn{2}{c|}{$\mathbf{L i G a S i O}_{\mathbf{4}}$} & \multicolumn{2}{c|}{$\mathbf{L i G a}_{\mathbf{5}} \mathbf{O}_{\mathbf{8}}$} & \multicolumn{2}{c|}{$\boldsymbol{\gamma} \mathbf{- L i}_{\mathbf{5}} \mathbf{G a S i}_{\mathbf{2}} \mathbf{O}_{\mathbf{8}}$} \\
\hline $\mathrm{d}(\mathrm{\AA})$ & $\mathrm{I}(\%)$ & $\mathrm{d}(\mathrm{\AA})$ & $\mathrm{I}(\%)$ & $\mathrm{d}(\mathrm{A})$ & $\mathrm{I}(\%)$ & $\mathrm{d}(\mathrm{A})$ & $\mathrm{I}(\%)$ \\
\hline 3,999 & 7 & 4,000 & 100 & & & & \\
\hline 3,893 & 6 & 3,930 & 25 & & & 3,890 & 75 \\
\hline 3,644 & 12 & & & & & 3,647 & 70 \\
\hline 2,886 & 34 & & & 2,900 & 41 & & \\
\hline 2,693 & 29 & & & & & 2,688 & 100 \\
\hline 2,585 & 13 & 2,576 & 60 & & & 2,612 & 80 \\
\hline 2,463 & 100 & & & 2,473 & 100 & & \\
\hline 1,447 & 37 & & & 1,450 & 23 & 1,452 & 15 \\
\hline
\end{tabular}

Com os valores das intensidades relativas obtidos na difração de raios $\mathrm{X}$ fizemos uma estimativa da quantidade de cada uma das fases formadas nas amostras 1 e 2 . Na amostra 1, como podemos ver na Tabela 1 , a linha de difração mais intensa medida $(\mathrm{I} \%=100)$ é aquela mais intensa do $\mathrm{LiGa}_{5} \mathrm{O}_{8}$, segundo os dados JCPDS. Portanto este composto é a fase predominante na mistura. Esta linha, com $d=2,463 \AA$, foi tomada como referência para uma estimativa da composição relativa das fases remanescentes. A linha mais intensa do $\mathrm{LiGaSiO}_{4}$ segundo a base JCPDS corresponde à linha de intensidade $\mathrm{I}=7 \%$ com $d=3,999 \AA$ e aquela mais intensa do $\gamma-\mathrm{Li}_{5} \mathrm{GaSi}_{2} \mathrm{O}_{8}, d=2,693 \AA$, corresponde à uma intensidade medida de $29 \%$. Fazendo a normalização, as quantidades estimadas das fases foram: $\mathrm{LiGa}_{5} \mathrm{O}_{8}$ com $74 \%$, seguido pelo $\gamma-\mathrm{Li}_{5} \mathrm{GaSi}_{2} \mathrm{O}_{8}$ com $24 \%$ e $\mathrm{LiGaSiO}_{4}$ com apenas $6 \%$.

Para a amostra 2, Tabela 2, a linha de difração mais intensa medida (I\% $=100)$, com $d=2,470 \AA$, que corresponde à linha de máximo de difração do $\mathrm{LiGa}_{5} \mathrm{O}_{8}$ foi tomada como referência, seguindo o procedimento anterior. A linha mais intensa do $\mathrm{LiGaSiO}_{4}$ tem intensidade $\mathrm{I}=34 \%$ em $d=4,000 \AA$ e aquela mais intensa do $\gamma-\mathrm{Li}_{5} \mathrm{GaSi}_{2} \mathrm{O}_{8}, d=2,700 \AA$, corresponde a uma intensidade medida de $24 \%$. As quantidades estimadas das fases foram: $\mathrm{LiGa}_{5} \mathrm{O}_{8}$ com $68 \%$, seguido pelo $\mathrm{LiGaSiO}_{4}$ com $22 \%$ e $\gamma-\mathrm{Li}_{5} \mathrm{GaSi}_{2} \mathrm{O}_{8}$ com $16 \%$.

Tabela 2: Linhas de difração mais intensas obtidas para a amostra 2.

\begin{tabular}{|c|c|c|c|c|c|c|c|}
\cline { 3 - 8 } \multicolumn{1}{c|}{} & \multicolumn{6}{c|}{ Valores Tabelados } \\
\hline \multicolumn{2}{|c|}{ Valores Medidos } & \multicolumn{2}{c|}{ LiGaSiO $_{\mathbf{4}}$} & \multicolumn{2}{c|}{$\mathbf{L i G a}_{\mathbf{5}} \mathbf{O}_{\mathbf{8}}$} & \multicolumn{2}{c|}{$\gamma \mathbf{- L i}_{\mathbf{5}} \mathbf{G a S i}_{\mathbf{2}} \mathbf{O}_{\mathbf{8}}$} \\
\hline $\mathrm{d}(\AA)$ & $\mathrm{I}(\%)$ & $\mathrm{d}(\AA)$ & $\mathrm{I}(\%)$ & $\mathrm{d}(\AA)$ & $\mathrm{I}(\%)$ & $\mathrm{d}(\AA)$ & $\mathrm{I}(\%)$ \\
\hline 4,000 & 34 & 4,000 & 100 & & & & \\
\hline 3,927 & 11 & 3,930 & 25 & & & 3,890 & 75 \\
\hline 3,660 & 14 & & & 3,669 & 17 & 3,635 & 70 \\
\hline 2,895 & 34 & & & 2,900 & 41 & & \\
\hline 2,766 & 25 & 2,769 & 63 & & & & \\
\hline 2,700 & 24 & & & & & 2,686 & 100 \\
\hline 2,574 & 26 & 2,576 & 60 & & & 2,565 & 25 \\
\hline 2,470 & 100 & & & 2,473 & 100 & & \\
\hline 1,577 & 23 & & & 1,578 & 23 & & \\
\hline 1,562 & 13 & 1,564 & 12 & & & 1,563 & 25 \\
\hline 1,449 & 37 & & & 1,450 & 23 & 1,452 & 15 \\
\hline
\end{tabular}

Estes resultados são mostrados nos histogramas da Figura 2 (a) e (b), para as amostras 1 e 2 , respectivamente. O método de preparo aumentou em $360 \%$ a quantidade da fase $\mathrm{LiGaSiO}_{4}$, da amostra $2 \mathrm{em}$ relação à amostra 1 , enquanto a quantidade do $\gamma-\mathrm{Li}_{5} \mathrm{GaSi}_{2} \mathrm{O}_{8}$ diminuiu em $66 \%$ e a do $\mathrm{LiGa}_{5} \mathrm{O}_{8}$ sofreu uma redução de apenas $8 \%$. Estes dados mostram que os compostos que tem silício em sua fórmula molecular têm a formação desfavorecida em relação ao composto que não o contém. 

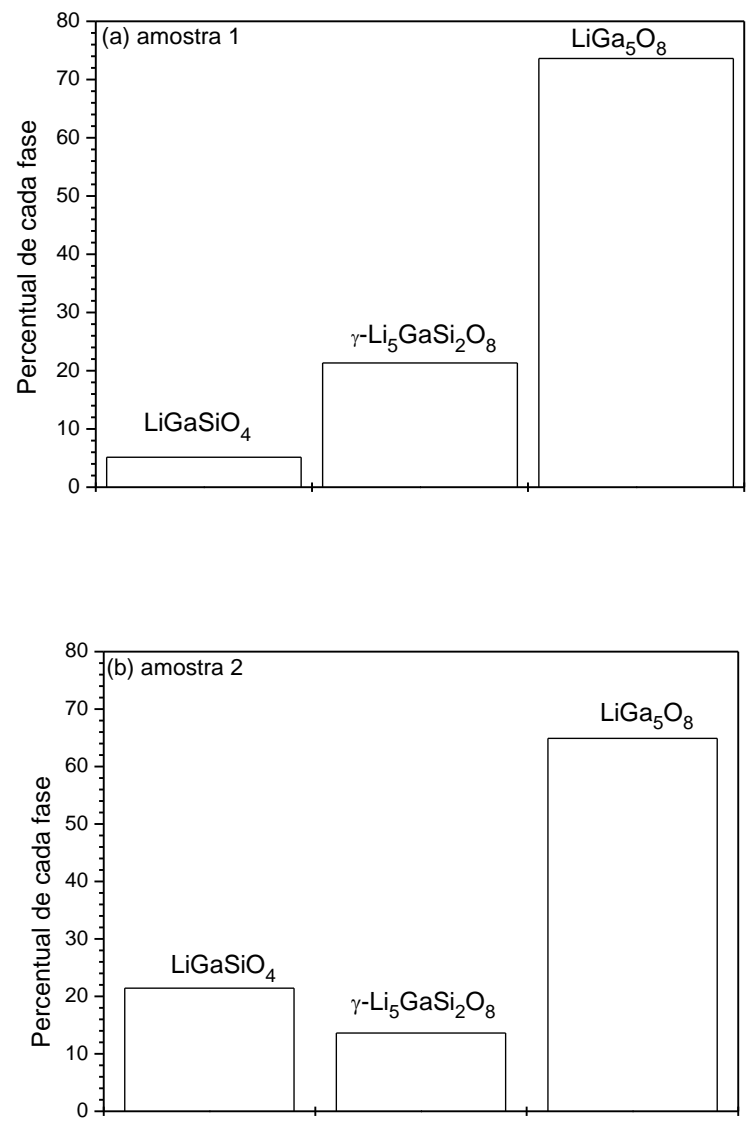

Figura 2: Histograma representando a proporção entre as fases formadas. (a) Na amostra 1, a fase predominante é o $\mathrm{LiGa}_{5} \mathrm{O}_{8}$, com $74 \%$, seguido pelo $\gamma-\mathrm{Li}_{5} \mathrm{GaSi}_{2} \mathrm{O}_{8}$ com $24 \%$ e $\mathrm{LiGaSiO}_{4}$ com apenas $6 \%$. (b) Amostra 2. A fase predominante ainda é o $\mathrm{LiGa}_{5} \mathrm{O} 8 \mathrm{com} 68 \%$, seguido pelo $\mathrm{LiGaSiO}_{4} \operatorname{com} 22 \%$ e $\gamma$ $\mathrm{Li}_{5} \mathrm{GaSi}_{2} \mathrm{O}_{8}$ com $16 \%$.

$\mathrm{O}$ silicato de lítio e gálio, $\mathrm{LiGaSiO}_{4}$ tem uma estrutura ordenada pertencente ao grupo espacial com simetria trigonal $R \overline{3}$, com parâmetros de célula unitária $a=13,635 \AA$ e $c=9,093 \AA$ [10], equivalente ao $\mathrm{LiAlGeO}_{4}$ [12], no qual todos os íons são coordenados tetraedricamente. Na estrutura, os íons $\mathrm{Ga}^{3+}$ ocupam posições chamadas $\mathrm{T} 1$, enquanto os íons $\mathrm{Li}^{+}$e $\mathrm{Si}^{4+}$ ocupam as posições denominadas $\mathrm{T} 2$ e $\mathrm{T} 3$ [7].

$\mathrm{O} \mathrm{LiGa}_{5} \mathrm{O}_{8}$ pertence a uma classe de materiais de fórmula $\mathrm{AB}_{2} \mathrm{O}_{4}$ chamados de espinélios, nos quais existem duas posições para os cátions $\mathrm{A}^{2+} \mathrm{e}^{3+}$. A célula unitária pertence ao grupo cúbico espacial $O_{h}^{7}(F d 3 m)$ com parâmetro de rede igual a 8,221 Å e contém 32 oxigênios com 96 interstícios, onde 32 destes são octaédricos (íons B) e os outros 64 são tetraédricos (íons A), com 8 metais divalentes e 16 metais trivalentes. No espinélio invertido, os sítios tetraédricos são ocupados por cations B e os sítios octaédricos são aleatoriamente ocupados por cátions $\mathrm{A}^{2+} \mathrm{e} \mathrm{B}^{3+}[13]$.

A estrutura do composto $\gamma-\mathrm{Li}_{5} \mathrm{GaSi}_{2} \mathrm{O}_{8}$ não é bem conhecida, mas parece ser derivada da rutila $\gamma$ $\mathrm{Li}_{3} \mathrm{PO}_{4}$, que possui simetria ortorrômbica, pertencente ao grupo espacial Pnma, composta por um arranjo hexagonal de oxigênios, com os átomos de fósforo coordenados tetraedricamente [ $\underline{8}]$.

Nas fases formadas existem os cátions $\mathrm{Li}^{+}, \mathrm{Ga}^{3+}$ ou $\mathrm{Si}^{4+}$. Os raios iônicos do $\mathrm{Cr}^{3+}$ e do $\mathrm{Ga}^{3+}$ são, respectivamente $0,64 \AA$ e $0,62 \AA$, enquanto o $\mathrm{Li}^{+}$tem raio iônico de $0,68 \AA$ e o raio do $\mathrm{Si}^{4+}$ é igual a $0,42 \AA$. Pelos valores dos raios, seria mais provável a ocupação substitucional do $\mathrm{Cr}^{3+}$ no sítio do $\mathrm{Ga}^{3+}$ ou do $\mathrm{Li}^{+}$, pois como o raio iônico do $\mathrm{Si}^{4+}$ é 66 \% menor do que o do $\mathrm{Cr}^{3+}$, esta ocupação causaria uma grande desarrumação na rede hospedeira. Além disso, o mais importante requisito para reações entre óxidos é que o íon dopante tenha a valência igual àquela do íon que será substituído. Neste caso, é pouco provável que o $\mathrm{Cr}^{3+}$ substitua os íons de $\mathrm{Li}^{+}$ou de $\mathrm{Si}^{4+}$. Por isso acreditamos que a substituição mais conveniente seja a de íons de $\mathrm{Ga}^{3+}$ por íons 
de $\mathrm{Cr}^{3+}$ em todas as três fases, com três sítios tetraédricos e um sítio octaédrico (do $\mathrm{LiGa}_{5} \mathrm{O}_{8}$ ) disponíveis para a ocupação.

\subsection{Espectroscopia Fototérmica}

O objetivo principal na utilização da espectroscopia fototérmica é o fato de que esta técnica fornece o espectro de absorção óptica de compostos opacos dopados com metais de transição. O espectro apresenta formato e posição espectral da(s) banda(s) de absorção característica(s) do metal de transição dopante e da simetria do sítio de ocupação. Portanto, esta técnica pode responder as seguintes perguntas: a valência do cromo é realmente $3+$ ? O cromo está em sítio octaédrico ou tetraédrico? A primeira pergunta pode ser respondida também com a pura e simples observação da cor da amostra. A cor verde é característica da valência $3+$ do cromo, enquanto o $\mathrm{Cr}^{4+}$ é caracterizado por uma cor escura, quase marrom. As amostras produzidas neste trabalho apresentam cor verde, indicando a valência 3+ do cromo.

Nas Figuras 3 e 4 exibimos o espectro obtido paras as amostras 1 e 2, respectivamente. Comparando estes dados com resultados da literatura, concluímos que as posições espectrais e formatos das largas bandas observadas nas amostras 1 e 2 são característicos de transições do $\mathrm{Cr}^{3+}$ em sítios octaédricos [1-7,14-17]. Com isso, concluímos que o $\mathrm{Cr}^{3+}$ ocupa por substituição alguns sítios octaédricos do $\mathrm{Ga}^{3+}$ no $\mathrm{LiGa}_{5} \mathrm{O}_{8}$, já que entre as fases identificadas nas amostras apenas esta dispõe de sítios octaédricos.

$\mathrm{O}$ termo fundamental de energia do $\mathrm{Cr}^{3+}$ livre é o ${ }^{4} F$ (configuração eletrônica $d^{3}$ ). Porém, quando o íon está ligado em uma rede cristalina, os seus estados de energia são desdobrados pelo campo eletrostático (chamado de campo cristalino) gerado pelos ânions ligantes. Este desdobramento dependerá da intensidade do campo cristalino, definida pelo parâmetro $D q$ e da repulsão intereletrônica, definida pelo parâmetro de Racah $B$. O termo ${ }^{4} F$ se desdobra, em ordem crescente de energia, nos estados ${ }^{4} A_{2}\left({ }^{4} F\right),{ }^{4} T_{2}\left({ }^{4} F\right)$ e ${ }^{4} T_{1}\left({ }^{4} F\right)$. O segundo termo de energia é o o ${ }^{4} P$, que se desdobra no estado ${ }^{4} T_{l}\left({ }^{4} P\right)$. Em seguida vem o termo ${ }^{2} G$, que se desdobra nos estados ${ }^{2} E\left({ }^{2} G\right),{ }^{2} T_{l}\left({ }^{2} G\right),{ }^{2} T_{2}\left({ }^{2} G\right)$ e ${ }^{2} A_{l}\left({ }^{2} G\right)$. O estado fundamental do $\mathrm{Cr}^{3+}$ é o ${ }^{4} A_{2}\left({ }^{4} F\right)$. Para valores $D q / B<2,4$ o campo cristalino é fraco e o primeiro estado excitado é o ${ }^{4} T_{2}\left({ }^{4} F\right)$. Para campos cristalinos fortes, com $D q / B>$ 2,4, o primeiro estado excitado é o ${ }^{2} E\left({ }^{2} G\right)$. Os estados ${ }^{4} A_{2}\left({ }^{4} F\right)$ e ${ }^{2} E\left({ }^{2} G\right)$ tem configuração eletrônica $t_{2}^{3}$ enquanto os estados ${ }^{4} T_{2}\left({ }^{4} F\right)$ e ${ }^{4} T_{1}\left({ }^{4} F\right)$ tem configuração $t_{2}^{2} e$. Isto implica que transições entre os estados ${ }^{4} A_{2}\left({ }^{4} F\right)$ e ${ }^{2} E\left({ }^{2} G\right)$ originam bandas estreitas no espectro, enquanto transições entre os estados ${ }^{4} A_{2}\left({ }^{4} F\right)$ e ${ }^{4} T_{2}\left({ }^{4} F\right)$ ou ${ }^{4} A_{2}\left({ }^{4} F\right)$ e ${ }^{4} T_{l}\left({ }^{4} F\right)$ originam bandas largas $[\underline{14}, \underline{18}]$. Por isto associamos as bandas observadas à transições entre o estado fundamental ${ }^{4} A_{2}\left({ }^{4} F\right)$ e os estados excitados ${ }^{4} T_{2}\left({ }^{4} F\right)$ e ${ }^{4} T_{1}\left({ }^{4} F\right)$.

$\mathrm{O}$ espectro da amostra 1, Figura 3, apresenta duas bandas. A de maior energia, com baricentro em 413 $\mathrm{nm}\left(24213 \mathrm{~cm}^{-1}\right)$ é atribuída à transição ${ }^{4} A_{2}\left({ }^{4} F\right) \rightarrow{ }^{4} T_{1}\left({ }^{4} F\right)$. A banda de menor energia, com máximo de intensidade em $603 \mathrm{~nm}\left(16583 \mathrm{~cm}^{-1}\right)$ é atribuída à transição ${ }^{4} A_{2}\left({ }^{4} F\right) \rightarrow{ }^{4} T_{2}\left({ }^{4} F\right)$. As duas transições são permitidas por spin, originando por isso bandas intensas.

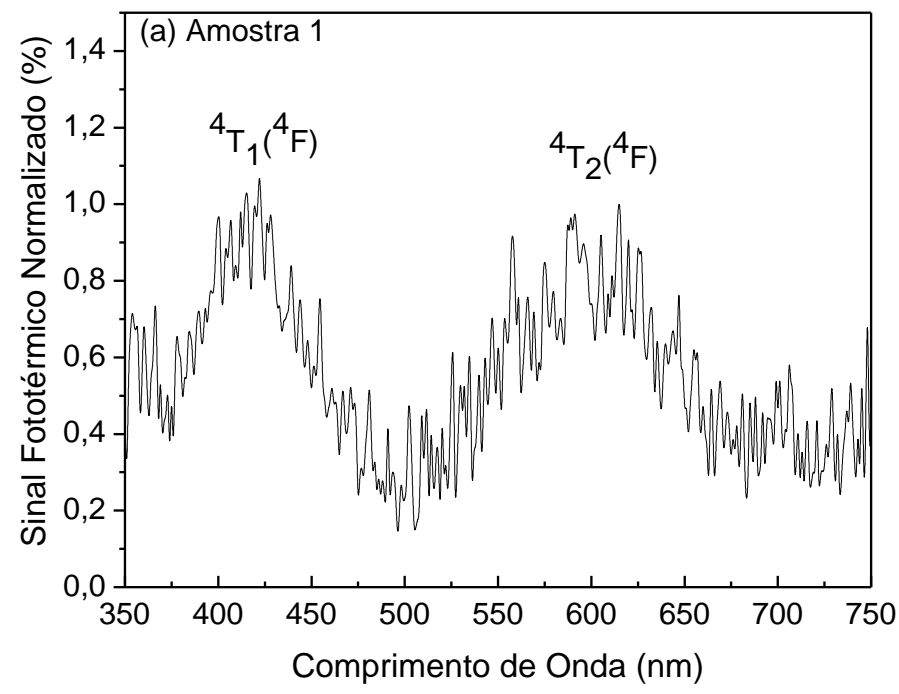

Figura 3: Espectro fototérmico da amostra 1, obtido à temperatura ambiente. 
Para a amostra 2 foi obtido o espectro de absorção exibido na Figura 4. Podemos observar duas bandas, com baricentro em $411 \mathrm{~nm}\left(24331 \mathrm{~cm}^{-1}\right)$ e $587 \mathrm{~nm}\left(17035 \mathrm{~cm}^{-1}\right)$, atribuídas às transições do $\mathrm{Cr}^{3+}$, ${ }^{4} A_{2}\left({ }^{4} F\right) \rightarrow{ }^{4} T_{1}\left({ }^{4} F\right)$ e ${ }^{4} A_{2}\left({ }^{4} F\right) \rightarrow{ }^{4} T_{2}\left({ }^{4} F\right)$, respectivamente. O espectro observado, como o da amostra 1 , apresenta o formato característico de transições de energia do $\mathrm{Cr}^{3+}$ em sítio de coordenação octaédrica.



Figura 4: Espectro fototérmico da amostra 2 obtido à temperatura ambiente.

Os valores de $D q$ e $B$ são encontrados pelas posições de energia do máximo de intensidade das bandas ${ }^{4} T_{2}\left({ }^{4} F\right)$ e ${ }^{4} T_{1}\left({ }^{4} F\right)$ respectivamente. Com esses valores de energia e com as matrizes de Tanabe-Sugano para a configuração $d^{3}[\underline{14}, \underline{18}]$ resolvidas e apresentadas nas equações 1 e 2 a seguir, os parâmetros foram calculados e estão relacionados nas Tabelas 3 e 4 .

$$
\begin{aligned}
& { }^{4} A_{2}\left({ }^{4} F\right) \rightarrow{ }^{4} T_{2}\left({ }^{4} F\right)=10 D q \\
& B=\left[\frac{(\Delta E / D q)^{2}-10(\Delta E / D q)}{15(\Delta E / D q-8)}\right] D q
\end{aligned}
$$

onde $\Delta E$ é a diferença de energia dos níveis ${ }^{4} T_{2}\left({ }^{4} F\right)$ e ${ }^{4} T_{1}\left({ }^{4} F\right)$.

\begin{tabular}{|c|c|c|c|}
\hline Transição & Comprimento de onda (nm) & Energia $\left(\mathrm{cm}^{-1}\right)$ & Parâmetros de energia $\left(\mathrm{cm}^{-1}\right)$ \\
\hline${ }^{4} A_{2}\left({ }^{4} F\right) \rightarrow{ }^{4} T_{1}\left({ }^{4} F\right)$ & 413 & 24213 & \multirow{2}{*}{$\begin{aligned} D q & =1658 \\
B & =807\end{aligned}$} \\
\hline${ }^{4} A_{2}\left({ }^{4} F\right) \rightarrow{ }^{4} T_{2}\left({ }^{4} F\right)$ & 603 & 16583 & \\
\hline
\end{tabular}

Tabela 3: Posições das transições em comprimento de onda e em energia da amostra 1.

Tabela 4: Posições das transições em comprimento de onda e em energia da amostra 2.

\begin{tabular}{|c|c|c|c|}
\hline Transição & Comprimento de onda (nm) & Energia $\left(\mathbf{c m}^{-1}\right)$ & Parâmetros de energia $\left(\mathbf{c m}^{-1}\right)$ \\
\hline${ }^{4} A_{2}\left({ }^{4} F\right) \rightarrow{ }^{4} T_{1}\left({ }^{4} F\right)$ & 411 & 24331 & $D q=1703$ \\
${ }^{4} A_{2}\left({ }^{4} F\right) \rightarrow{ }^{4} T_{2}\left({ }^{4} F\right)$ & 587 & 17035 & $B=746$ \\
\hline
\end{tabular}


Os parâmetros de campo cristalino e de Racah obtidos para a amostra 1 são $D q=1658 \mathrm{~cm}^{-1}$ e $B=$ $807 \mathrm{~cm}^{-1}$. Os mesmos parâmetros, calculados para os íons $\mathrm{Cr}^{3+}$ ocupando os sítios da amostra 2 são, respectivamente, $1703 \mathrm{~cm}^{-1}$ e $746 \mathrm{~cm}^{-1}$. Os valores encontrados para o parâmetro $D q$ são característicos do $\mathrm{Cr}^{3+}$ em sítio de ocupação octaédrico. Pode ser verificado que a razão $D q / B$ para a amostra 1 é 2,2 e para a amostra $2, D q / B=2,4$. Sistemas com $D q / B \approx 2,3$ apresentam campo cristalino intermediário, onde os níveis de energia ${ }^{4} T_{2}\left({ }^{4} F\right)$ e ${ }^{2} E\left({ }^{2} G\right)$ estão muito próximos, ocorrendo uma mistura de orbitais. Porém os valores $D q / B$, junto com os formatos das bandas, indicam que o primeiro estado excitado é realmente o ${ }^{4} T_{2}\left({ }^{4} F\right)$ [14-17]. A ocorrência da transição $\left.{ }^{2} A_{2}\left({ }^{4} F\right) \rightarrow{ }^{4} T_{1}{ }^{4} P\right)$ é esperada na região abaixo de $400 \mathrm{~nm}$. Entretanto, esta banda não foi observada porque a instrumentação disponível não responde nesta região espectral. Os íons $\mathrm{Cr}^{3+}$ estão mais comprimidos nos sítios da amostra 2, pois o parâmetro $D q$ desta amostra é maior do que o campo cristalino da amostra 1. Além disso a redução do parâmetro de Racah $B$ indica a maior covalência da ligação impurezaligante na amostra 2, em comparação com a amostra 1 . $\mathrm{O}$ formato das bandas indica que existe apenas um sítio octaédrico em cada amostra. $\mathrm{O}$ fato do íon $\mathrm{Cr}^{3+}$ ter entrado por substituição no sítio do $\mathrm{Ga}^{3+} \mathrm{do} \mathrm{LiGa}_{5} \mathrm{O}_{8}$ demonstra a grande afinidade destes íons com os sítios octaédricos, em detrimento dos sítios tetraédricos das fases $\mathrm{LiGaSiO}_{4}$ e $\gamma-\mathrm{Li}_{5} \mathrm{GaSi}_{2} \mathrm{O}_{8}$ e do próprio $\mathrm{LiGa}_{5} \mathrm{O}_{8}$.

\section{CONCLUSÃO}

Os dados de difração de raios $\mathrm{X}$ mostram a formação das fases, $\mathrm{LiGaSiO}_{4}, \mathrm{LiGa}_{5} \mathrm{O}_{8}$ e $\gamma-\mathrm{Li}_{5} \mathrm{GaSi}_{2} \mathrm{O}_{8}$, sendo notada uma grande variação na quantidade de $\mathrm{LiGaSiO}_{4}$ em função do método de preparo. Os espectros fototérmicos das amostras à temperatura ambiente exibem bandas largas, atribuídas às transições permitidas por spin ${ }^{4} A_{2}\left({ }^{4} F\right) \rightarrow{ }^{4} T_{1}\left({ }^{4} F\right)$ e ${ }^{4} A_{2}\left({ }^{4} F\right) \rightarrow{ }^{4} T_{2}\left({ }^{4} F\right)$ do $\mathrm{Cr}^{3+}$ em sítio octaédrico. Com os valores das posições de energia dos baricentros das bandas e com as matrizes de Tanabe-Sugano, obtivemos os parâmetros de campo cristalino $D q$ e de Racah $B$. Para a amostra $1, D q=1658 \mathrm{~cm}^{-1}$ e $B=807 \mathrm{~cm}^{-1}$ e para a amostra $2, D q=1703$ $\mathrm{cm}^{-1}$ e $B=746 \mathrm{~cm}^{-1}$, característicos do $\mathrm{Cr}^{3+}$ octaédrico. Os valores de $D q / B$ indicam que o primeiro estado excitado é o ${ }^{4} T_{2}\left({ }^{4} F\right)$. Embora existam sítios tetraédricos nas fases formadas, não verificamos a ocupação do $\mathrm{Cr}^{3+}$ neste tipo de sítio. Isto pode significar uma grande preferência dos íons $\mathrm{Cr}^{3+}$ pelos sítios octaédricos. Ou também a geração de um sinal muito fraco devido à baixa ocupação dos sítios tetraédricos pelo $\mathrm{Cr}^{3+}$, que assim ficaria sobreposto pelo sinal mais intenso gerado pelo $\mathrm{Cr}^{3+}$ em sítio octaédrico.

\section{AGRADECIMENTOS}

As autoras agradecem a FAPERJ e CNPq pelo apoio financeiro, ao prof. H. Amorim, do IF/UFRJ, e ao prof. M. Massunaga, do LCFIS/UENF, pela colaboração.

\section{BIBLIOGRAFIA}

[1] KÜCK, S., "Laser-related spectroscopy of ion-doped crystals for tunable solid-state lasers", Applied Physics B: Lasers and Optics, v. 72, n. 5, pp. 515-562, April 2001.

[2] LAI, S.T., "Highly efficient emerald laser", Journal of Optical Society of American B, v. 4, n. 8, pp. 12861290, Aug. 1987.

[3] PAYNE, S.A., CHASE, L.L., NEWKIRK, H.W. et al., " $\mathrm{LiCaAlF}_{6}: \mathrm{Cr}^{3+}$ : A promising new solid state laser material", IEEE Journal of Quantum Electronics, v. 24, n.11, pp. 2243-2252, Nov. 1988.

[4] STALDER, M., CHAI, B.H.T., BASS, M., “The flashlamp-pumped Cr: LiSrAlF6 laser”, Applied Physics Letters, v. 58, n. 3, pp. 216-218, Jan. 1991.

[5] LAI, S.T, CHAI, B.H.T., LONG, M., et al., "Room-temperature near-infrared tunable Cr-La $\mathrm{Ga}_{5} \mathrm{SiO}_{14}$ laser”, IEEE Journal of Quantum Electronics, v. 24, n. 9, 1922-1926, Sep.1988.

[6] YAMAGA, M., WELLS, J.P.R., HONDA, M., et al., "Investigation on the valence of Cr Ions in $\mathrm{LiAlO}_{2}$ " Journal of Luminescence, v. 108, n. 1-4, pp. 313-317, Jun. 2004.

[7] VEREMECHIK, T.F., ZHARIKOV, E.V., SUBBATIN, K.K., "New laser crystals of complex oxides doped with ions of $d$ elements with variable valence and different structural localizations review", Crystallography Reports, v. 48, n. 6, pp. 974-988, Nov. 2003. 
[8] QUINTANA, P., WEST, A.R., "Compound formation and phase equilibria in the system $\mathrm{Li}_{4} \mathrm{SiO}_{4}$ $\mathrm{LiGaSiO}_{4}$ ”, Journal of Solid State Chemistry, v. 81, pp. 257-270, Aug. 1989.

[9] KITTEL, C., Introdução à Física do Estado Sólido, Rio de Janeiro, Guanabara Dois, 1996.

[10] JCPDS-ICDD, 26-846, 1996.

[11] JCPDS-ICDD, 38-1371, 1996.

[12] MURAYAMA, M., KANNO, R., KAWAMOTO, Y., et al., "Structure of the Thio-LISICON-Li $\mathrm{GeS}_{4}$ ", Solid States Ionics, v. 154-155, pp. 799-794, Dec. 2002.

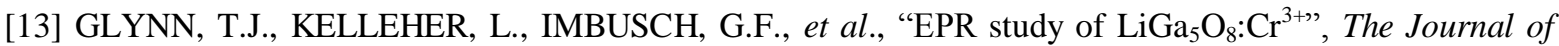
Chemical Physics, v. 55, n. 6, pp. 2925-2930, Sep. 1971.

[14] HENDERSON, B., IMBUSCH, G.F., Optical Spectroscopy of Inorganic Solids, Oxford, Clarendon, 1989.

[15] GRINBERG, M., SIKORSKA, A., SLIWINSKII, A., et al., "Photoacoustic and optical absorption spectroscopy studies of luminescent $\mathrm{Cr}^{3+}$ and $\mathrm{Cr}^{4+}$ centers in yttrium aluminum garnet", Physical Review B, v. 67, pp. 045113-1-045113-9, Jan. 2003.

[16] SOSMAN, L.P., ABRITTA, T., NAKAMURA, et al., "Luminescence and photoacoustic study of $\mathrm{MgGa}_{2} \mathrm{O}_{4}: \mathrm{Cr}^{3+}$, , Physica Status Solidi A-Applied Research, v. 147, pp. k107-110, Feb. 1995.

[17] DEREŃ, P.J., MALINOWISKI, M., STRĘK, W., "Site selection spectroscopy of $\mathrm{Cr}^{3+}$ In $\mathrm{MgAl}_{2} \mathrm{O}_{4} \mathrm{green}$ spinel”, Journal of Luminescence, v. 68, n. 1-4, pp. 91-103 May 1996.

[18] TANABE, Y., SUGANO, S., "On the absorption of complex ions", Journal of the Physical Society of Japan, v. 9, n. 5, pp. 753-779, 1954. 